\title{
O papel da medicina de família nos cuidados paliativos
}

\author{
Diego Bonaparte Martins ${ }^{1}$ \\ Marcelo Marcos Piva Demarzo ${ }^{1}$
}

\section{RESUMO}

Introdução: O Brasil está entre os países nos quais a expectativa de vida aumentou consideralmente nas últimas décadas, com uma proporção cada vez maior de pessoas acima de 60 anos, e nesse contexto os cuidados paliativos na Atenção Primária à Saúde (APS) se configuram numa necessidade em saúde. Fatores como a idade avançada ou a presença de doenças crônicodegenerativas não transmissíveis, elegem o paciente a receber os cuidados paliativos, visto que, proporcionam a minimização da dor e trazem mais qualidade de vida ao paciente e à família. No entanto, a realidade brasileira quanto à formação pósgraduada de Médicos de Família, principal especialidade médica da APS, ainda necessita de expansão e mais quaificação para atender à essa necessidade crescente. A carência de políticas públicas de saúde e uma constante revisão do currículo acadêmico são questões a serem discutidas, visando a busca por uma formação mais qualificada para o futuro Médico de Família. Método: Estudo desenvolvido por meio de revisão bibliográfica narrativa exploratória tradicional. Resultados: Existe a necessidade de formarmos mais Médicos de Família para assistirem os eleitos a receberem os cuidados paliativos, em paralelo, é preciso trabalharmos pela criação de novas políticas públicas de saúde, aliadas à revisão constante das disciplinas que integram o currículo acadêmico. A vivência e o conhecimento, inicialmente por meio dos estágios, agregarão à formação do Médico de Família, levando-o a desempenhar suas atividades junto à Atenção Primária à Saúde, por meio de cuidado paliativo, adequadamente. Considerações Finais e Conclusão: Atualmente, os recursos humanos que possuimos são poucos e há urgência por uma revisão no currículo acadêmico, como também na estrutura de Atenção Primária à Saúde para oferecer o cuidado paliativo, levando dignidade ao paciente no fim da vida. O caminho mais rápido para levarmos os cuidados paliativos à todos os pacientes eleitos, é por meio da criação de consistentes políticas públicas de saúde.

Cuidados Paliativos, Medicina de Família, Atenção Primária à Saúde

${ }^{1}$ Faculdade São Leopoldo Mandic, Curso de Medicina, Programa de Pós-Graduação Residência Medicina de Família. Rua José Rocha Junqueira, 13, 13045-755, Campinas, SP, Brasil. Correspondência para: DB Martins. E-mail: < diegobonaparte@gmail.com>.

${ }^{2}$ Faculdade São Leopoldo Mandic, Curso de Medicina, Departamento de Atenção Primária à Saúde. Campinas, SP, Brasil. 


\section{ABSTRACT}

Introduction: Brazil is among the countries in which life expectancy has increased considerably in recent decades, with an increasing proportion of people over 60 years of age, and in this context palliative care in Primary Health Care (PHC) is a health need. Factors such as advanced age or the presence of chronic-degenerative non-communicable diseases elect the patient to receive palliative care, since they provide pain minimization and bring more quality of life to the patient and family. However, the Brazilian reality regarding the postgraduate training of Family Physicians, the main medical specialty of PHC, still needs expansion and more qualification to meet this growing need. The lack of public health policies and a constant review of the academic curriculum are issues to be discussed, aiming at the search for a more qualified training for the future Family Doctor. Method: Study developed through traditional exploratory narrative bibliographic review. Results: There is a need to train more Family Doctors to assist elected officials to receive palliative care, in parallel, we need to work for the creation of new public health policies, together with the constant review of the disciplines that make up the academic curriculum. The experience and knowledge, initially through the internships, will add to the formation of the Family Doctor, leading him to perform his activities with Primary Health Care, through palliative care, properly. Final Considerations and Conclusion: Currently, the human resources we have are few and there is urgency for a review in the academic curriculum, as well as in the structure of Primary Health Care to offer palliative care, bringing dignity to the patient at the end of life. The fastest way to bring palliative care to all elected patients is through the creation of consistent public health policies.

\section{RESUMEN}

Introducción: Brasil se encuentra entre los países en los que la esperanza de vida ha aumentado considerablemente en las últimas décadas, con una proporción cada vez mayor de personas mayores de 60 años, y en este contexto la atención paliativa en la Atención Primaria de Salud (PHC) es una necesidad de salud. Factores como la edad avanzada o la presencia de enfermedades crónico degenerativas no transmisibles eligen al paciente para recibir cuidados paliativos, ya que proporcionan minimización del dolor y aportan más calidad de vida al paciente y a la familia. Sin embargo, la realidad brasileña con respecto a la formación de postgrado de Médicos de Familia, la principal especialidad médica de la PHC, todavía necesita expansión y más cualificación para satisfacer esta creciente necesidad. La falta de políticas de salud pública y una revisión constante del currículo académico son temas a discutir, con el objetivo de buscar una formación más cualificada para el futuro Médico de Familia. Método: Estudio desarrollado a través de la revisión bibliográfica narrativa exploratoria tradicional. Resultados: Es necesario capacitar a más Médicos de Familia para ayudar a los funcionarios electos a recibir cuidados paliativos, en paralelo, tenemos que trabajar para la creación de nuevas políticas de salud pública, junto con la revisión constante de las disciplinas que componen el currículo académico. La experiencia y el conocimiento, inicialmente a través de las prácticas, se sumarán a la formación del Médico de Familia, lo que le llevará a realizar sus actividades con La Atención Primaria de Salud, a través de cuidados paliativos, adecuadamente. Consideraciones Finales y Conclusión: Actualmente, los recursos humanos que tenemos son escasos y hay urgencia para una revisión en el currículo académico, así como en la estructura de la Atención Primaria de Salud para ofrecer cuidados paliativos, aportando dignidad al paciente al final de la vida. La forma más rápida de llevar cuidados paliativos a todos los pacientes electos es mediante la creación de políticas de salud pública coherentes.

Palliative Care, Family Medicine, Primary Health Care.

\section{INTRODUÇÃO}

O Brasil vislumbra um cenário onde o progressivo envelhecimento da população, para as próximas décadas, é confirmado por meio de estudo do perfil sociodemográfico. O estudo de Indicadores Socidemográficos Prospectivos para o período de 1991 a 2030, nos traz a perspectiva de crescimento da população idosa e com ela, consequentemente, teremos um alavancamento das doenças crônicas-degenerativas [1-2].

A partir da Constituição Federal de 1998 instalou-se a proposta do Sistema Único de Saúde (SUS) que garante ao cidadão brasileiro o direito à saúde. Ao longo de décadas com o apoio e investimentos em pesquisas foi possível à ciência, aliada à tecnologia, que desenvolvesse procedimentos, tratamentos e medicamentos ampliando a expectativa de vida e até a cura de algumas doenças até então consideradas letais, em um passado não tão distante [3-4].

Há mais de três décadas, o Brasil possui dois sistemas 
de saúde, o SUS e o setor privado. Em 1981, o Ministério de Educação (MEC) reconhece a Medicina de Família e Comunidade como especialização, inicialmente com o nome de Medicina Geral Comunitária (MGC), e assim, o profissional com especialização passa a ser absorvido pelo SUS, no entanto, em breve o MF será levado para o setor privado, em virtude da grande comercialização de planos e seguros de saúde. Devido à pequena quantidade de profissionais qualificados, temos aqui, mais um motivo para o currículo acadêmico ser revisado frequentemente, e desta maneira, ampliaremos o número de profissionais preparados, inclusive com conhecimento quanto às inovações tecnológicas [5].

Reiteramos nossa compreensão quanto à ampliação da incidência e prevalência de condições crônicas, em especial das doenças crônico-degenerativas não transmissíveis (DCNT), dar-se também, como uma consequência da longividade, e norteados nesta constatação, afirmamos a necessidade de trabalharmos pela evolução da medicina, pois hoje ela não pode limitarse a ser tecnicista e biologista, focando somente na doença e não no paciente.

Há a necessidade de trabalharmos por uma medicina que veja o paciente de forma global, que busque a cada dia, ser mais humanizada. É essencial valorizarmos o aprimoramento do currículo acadêmico, com a inclusão de disciplinas que preparem o futuro profissional da saúde, para uma relação interpessoal com o paciente, familiares e demais profissionais que compõem a equipe multidisciplinar de saúde, visto que, é possível que outros sintomas sejam identificados, e esta equipe integrará o acompanhamento do paciente [6].

Os Cuidados Paliativos (CP) são parte primordial na Atenção Primária à Saúde (APS) onde proporcionam a minimização da dor, o conforto e uma melhor qualidade de vida ao paciente e à família, que também deve ser assistida. Neste momento onde o final da vida está próximo, seja por dias, semanas ou meses, a situação desistabiliza não só emocionalmente mas também, psíquica, espiritual e financeiramente. O abalo é forte e abrangente, e é neste momento, onde a medicina humanizada faz a diferença para todos os envolvidos, seja através da comunicação de más notícias de forma adequada, seja no trato diário com respeito, levando dignidade ao paciente em seus momentos finais [7-9].

Visando trabalhar por melhorias no seguimento do CP na APS, em 2015, foi elaborado um Currículo Baseado nas Competências $(C B C)$ pela Sociedade Brasileira de
Medicina de Família e Comunidade (SBMFC) onde os programas de residência médica foram comtemplados com mais conhecimentos. Vale destacar a necessidade de mantermos um olhar crítico para as inovações, de forma que, possamos levá-las aos pacientes contemplados com o CP por meio da APS, no entanto, oferecer O CP é desafiador para o SUS, porém deve chegar à todos os pacientes identificados com a necessidade. O Brasil não é o único país com uma grande população com idade mais avançada, e a situação ocorre em todos os países onde os idosos aumentam a cada dia [9-11].

Nas últimas décadas a Medicina de Família e Comunidade (MFC) surgiu como campo discursivo de grande importância junto aos módulos de APS, e desta forma, o Médico de Família (MF) é reconhecido socialmente, como o profissional que prioriza a relação interpessoal com o paciente e familiares, caracterizandose como facilitador ao acesso ao $\mathrm{CP}$, onde ocorrerá o estreitamento na relação com o paciente e familiares $[12,13]$

O Brasil está longe de ter estrutura adequada e recursos humanos qualificados para atender as necessidades de APS e CP, para desempenhar suas funções reais, minimizando as dificuldades tanto do paciente que esteja no fim da vida, quanto da família. Existe uma carência de políticas públicas de saúde específicas ao CP na APS, e por conseguinte o paciente muitas vezes é contemplado tardiamente $[14,15]$.

Este estudo visa analisar, compreender e discutir as competências quanto aos CP na APS, e desta forma promover o desenvolvimento e implantação de ações em prol de sanarmos os entraves da questão no Brasil.

\section{MÉTODOS}

A opção metodológica deste estudo foi por uma revisão bibliográfica narrativa, por se tratar de uma revisão exploratória tradicional, já que na revisão bibliográfica narrativa, não há necessariamente a definição de critérios rigorosos e explícitos na seleção dos artigos, não existindo uma preocupação em esgotar as fontes de informação $[16,17]$

Os artigos de revisão narrativa se tornam publicações amplas apropriadas para a descrição e discussão do desenvolvimento de um determinado assunto, sob o ponto de vista teórico e/ou contextual.

Cabe citar que esta categoria de artigos tem um papel fundamental para o profissional da saúde, pois permite 
adquirir conhecimento de forma a ampliar sua formação acadêmica, e atualizar-se com as políticas públicas disponíveis e conhecimento sobre um tema específico em um curto tempo, porém esses artigos com essa opção metodológica não fornecem respostas quantitativas para questões específicas [18].

A busca por literatura para o presente estudo, deuse no período de março de 2010 a dezembro de 2020, sendo realizada através de consultas na Biblioteca Virtual em Saúde (BVS) onde foram encontrados artigos das bases de dados Literatura Latino-Americana e do Caribe em Ciências da Saúde (LILACS), Scientific Electronic Library Online (SCIELO) e Biblioteca Virtual da Revista Brasileira de Medicina de Família e Comunidade (RBMFC) onde foram selecionadas as publicações dos últimos 10 anos.

Os descritores utilizados na busca foram "Cuidados Paliatvos", "Medicina de Família" e "Atenção Primária" seguido pelo uso dos seguintes filtros: texto completo, assunto principal: Cuidados Paliativos e idioma português. É importante pontuar que além dos filtros selecionados na base da BVS não houveram qualquer outro critério específico de inclusão de artigos para estudo, apenas foram determinados alguns critérios de exclusão como: artigos pagos, artigos em duplicação, e artigos como foco que divergente do objetivo principal do estudo.

\section{RESULTADOS}

Inicialmente identificamos 61 na base LILACS, 335 na base SCIELO e 435 na RBMFC. Trabalhando com os descritores "Cuidados Paliatvos", "Medicina de Família" e "Atenção Primária" identificamos 831 artigos.

$\mathrm{Na}$ sequência, através do filtro texto completo chegamos a 482 artigos, com o filtro principal cuidados paliativos, o número de artigos selecionados baixou para 396, e com o filtro idioma português, identificamos 19 artigos, sendo que após uma análise criteriosa foram excluímos 05 artigos e desta forma, para a elaboração deste estudo foram utilizados 14 artigos relacionados no Quadro 1.

\section{Quadro 1. Artigos analisados para elaboração desta revisão de artigo}

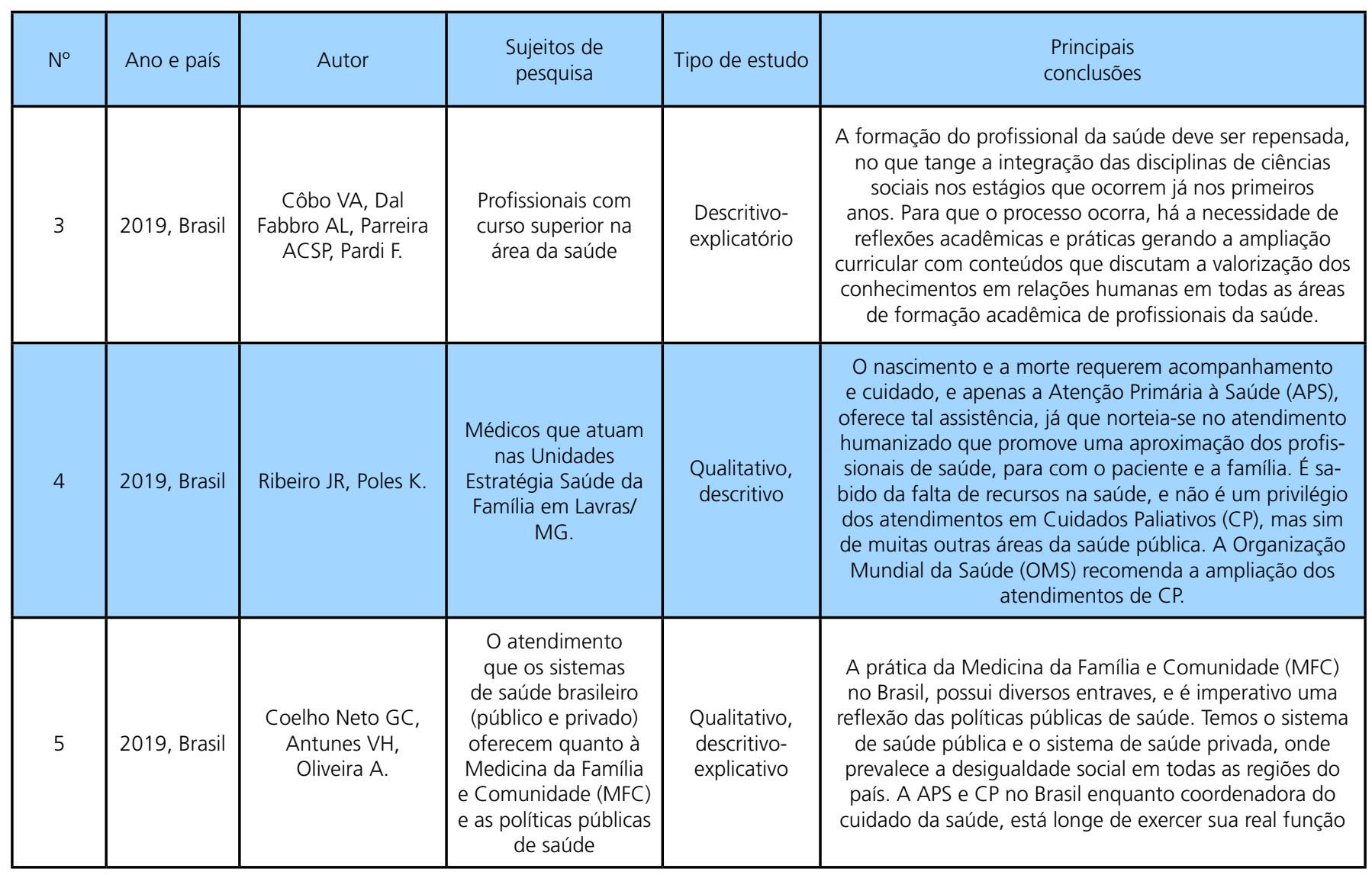




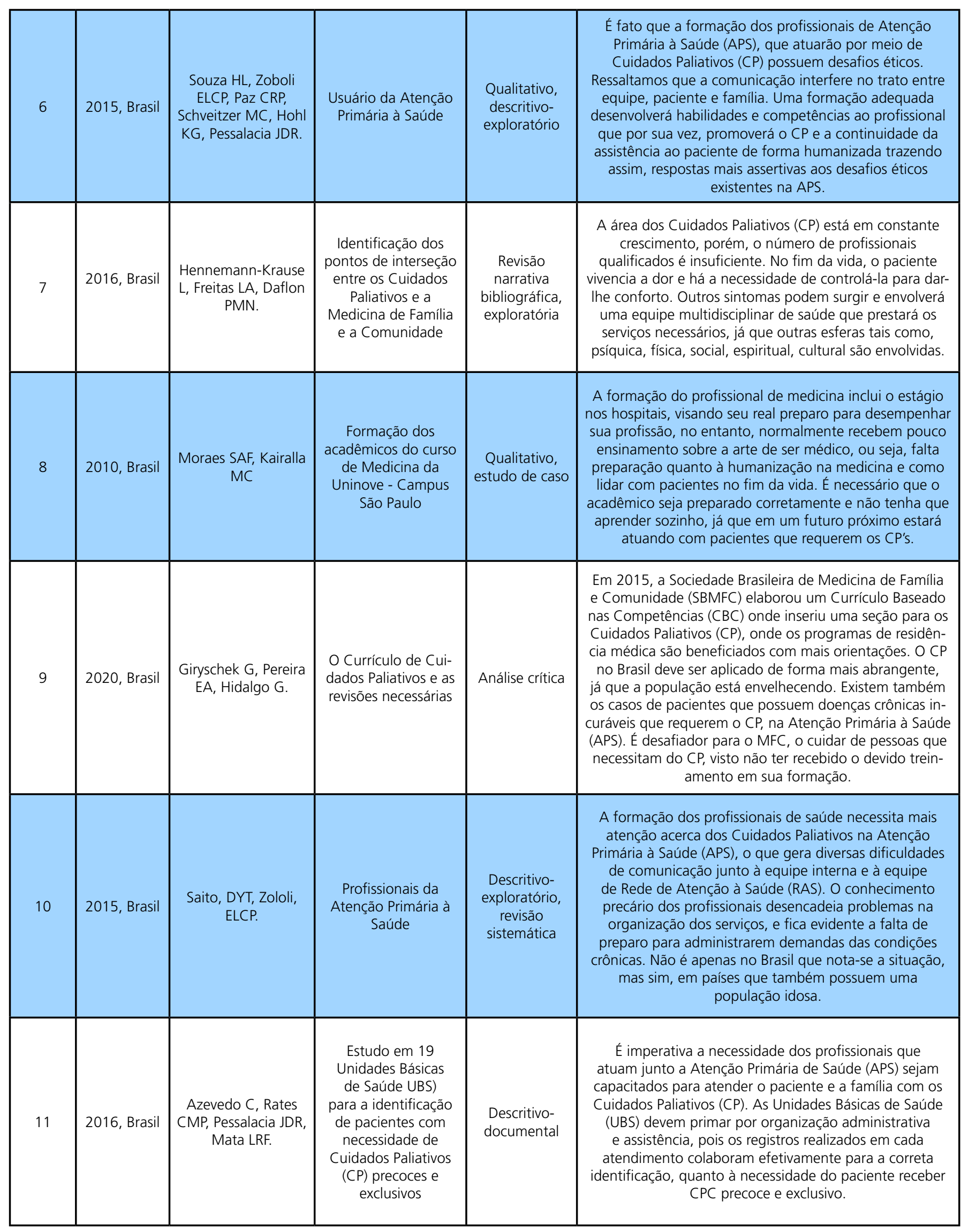




\begin{tabular}{|c|c|c|c|c|c|}
\hline 12 & 2018, Brasil & $\begin{array}{c}\text { Andrade HS, Alves } \\
\text { MGM, Carvalho SR, } \\
\text { Silva Júnior AG. }\end{array}$ & $\begin{array}{c}\text { O discurso dos } \\
\text { documentos que } \\
\text { amparam a Medicina } \\
\text { de Família e } \\
\text { Comunidade (MFC). }\end{array}$ & $\begin{array}{c}\text { Revisão } \\
\text { Bibliográfica }\end{array}$ & $\begin{array}{c}\text { Nas últimas décadas, a Medicina da Família e } \\
\text { Comunidade (MFC) destaca-se como prática e formação } \\
\text { discursiva na organização de modelo de Atenção Primária } \\
\text { à Saúde (APS). } \\
\text { Esta análise crítica pode nos levar à horizontes } \\
\text { promissores visando a produção de práticas clínicas que } \\
\text { qualifiquem, fortaleçam e defendam a APS no Brasil, } \\
\text { pensando no direito à saúde. }\end{array}$ \\
\hline 13 & 2014, Brasil & Silva, MLSR. & $\begin{array}{l}\text { Profissionais da } \\
\text { Atenção Primária à } \\
\text { Saúde }\end{array}$ & $\begin{array}{l}\text { Qualitativo, } \\
\text { descritivo- } \\
\text { exploratório }\end{array}$ & $\begin{array}{c}\text { Os profissionais da Atenção Primária à Saúde (APS) } \\
\text { acompanham o envelhecimento e a fragilização do } \\
\text { paciente, e é neste momento há a necessidade de } \\
\text { promover o cuidado não apenas ao paciente, mas } \\
\text { também assistir à família, que se depara com novas } \\
\text { situações, como o medo e a insegurança financeira. É } \\
\text { no desenvolvimento deste acolhimento que as ações da } \\
\text { Atenção Primária à Saúde e a Estratégia Saúde da Família } \\
\text { são evidenciadas e potencializadas. }\end{array}$ \\
\hline 14 & 2018, Brasil & $\begin{array}{c}\text { Ernesto Silva A, } \\
\text { Duarte ED. }\end{array}$ & $\begin{array}{l}\text { Profissionais } \\
\text { de Equipe } \\
\text { Multidisciplinar } \\
\text { do Atendimento } \\
\text { Domiciliar (EMAD) }\end{array}$ & $\begin{array}{l}\text { Qualitativo, } \\
\text { descritivo- } \\
\text { exploratório }\end{array}$ & $\begin{array}{c}\text { A Assistência Domiciliar (AD) é a atividade básica } \\
\text { desenvolvida pela Atenção Primária à Saúde (APS), } \\
\text { seja, temporariamente ou permanente. Uma equipe } \\
\text { multidisciplinar atua nos AD's e os pacientes são } \\
\text { selecionados por meio de critérios pré-estabelecidos pela } \\
\text { Rede de Atenção à Saúde (RAS). O Brasil oferece o AD } \\
\text { há } 20 \text { anos, no entanto, persiste a carência de políticas } \\
\text { públicas de saúde. }\end{array}$ \\
\hline
\end{tabular}

Fonte: Autoria própria, 2020.

\section{DISCUSSÃO}

É evidente que o crescimento da população idosa no Brasil nos remete à compreensão da necessidade de termos profissionais qualificados, através de um currículo estruturado, como também por meio da especiliazação em MFC, para que seja possível atendermos o paciente que tenha a necessidade de receber $\mathrm{O} C \mathrm{CP}$, acolhendo-o adequadamente, e principalmente de forma digna, já que é prioridade quando se pensa em levar qualidade de vida e conforto. No final da vida muitos fatores agregam, e levam o paciente a um quadro, quase sempre mais grave onde a presença da dor, do desconforto físico, emocional ou psíquico requer atenção. O MF, seja no SUS ou na rede privada precisa estar apto a levar ao paciente e à familia estratégias que promovam mais qualidade de vida com comunicação clara, no entanto, de forma humanizada.

A população brasileira está cada dia mais idosa e com a longividade, nos deparamos com uma crescente incidência das DCNT. É relevante trabalharmos por uma medicina mais humanizada, não apenas tecnicista, por meio da inclusão de disciplinas no currículo acadêmico, focando na qualificação do futuro profissional de saúde. Deste modo, o MF terá condições de relacionar-se com o paciente, com a equipe e com a família, de forma correta e segura. Estará apto a lidar com questões quanto às competências focadas no fim da vida, ou seja, emissão de atestados, administração da dor, preparação para a ocorrência do óbito, comunicação de más notícias e luto. Estas são algumas das situações que requerem qualificação, habilidade e trato ao desempenhá-las. A APS com suporte do CP pede um maior investimento e aprofundamento, tanto no currículo acadêmico do MF, como na formação dos demais profissionais da área de saúde, visto que, na saúde pública e na saúde privada há carência de profissionais com especialização em MFC, e os mesmos trabalham em conjunto a uma equipe multidisciplinar $[5,8,17]$.

O alívio do sofrimento do paciente pode ser ampliado, por meio da aplicação das tecnologias disponíveis e o correto envolvimento dos profissionais, utilizando-se de conhecimentos e habilidades adquiridos durante os 
estágios iniciais. Desta maneira, ao iniciarem a atuação profissional estarão aptos para um desempenho eficiente, eficaz e humanizado, já que vivenciaram diversas situações que contribuiram para sua qualificação profissional humanizada [3-9].

Sobretudo, sabemos que são poucos os especialistas para atuar na APS com os CP, e por este motivo os poucos especialistas são absorvidos pelo SUS, nota-se a probabilidade do setor privado de saúde intensificar a busca por MF, que anteriormente era um atendimento quase em sua totalidade realizado pelo SUS, salvo algumas exceções conforme o perfil do plano ou seguro de saúde contratado pelo paciente através do setor privado. Em suma, os profissionais capacitados para atuar com CP, aos poucos, estão migrando para o setor privado, o que leva a vislumbrar um possível colapso no SUS [13].

\section{Limitações do estudo}

Este estudo explicita a necessidade de propostas de políticas públicas na saúde, objetivando o atendimento de qualidade na APS por meio do CP, no entanto há limitações. A pesquisa norteou-se a partir da revisão bibliográfica de 14 artigos que propiciaram maior compreensão das variadas facetas da questão, como a necessidade de aprimoramento da grade curricular acadêmica dos profissionais da saúde, já que é um ponto de partida para a formação qualificada e humanizada do futuro profissional.

Hoje o MF só consegue esta qualificação por meio de uma especialização que proporciona mais conhecimento, informação e experiência para desempenhar suas atividades, levando dignidade ao paciente no fim da vida.

O Brasil ainda não possui a estrutura adequada com equipe de recursos humanos qualificados no atendimento de APS e CP, para suprimir a demanda do país, e assim levar ao paciente mais conforto no período final de sua vida. É eminente a necessidade de um programa conscistente de políticas públicas de saúde específicas ao CP na APS, instrumento primordial para oferecer $\mathrm{o}$ atendimento ideal.

\section{CONSIDERAÇÕES FINAIS E CONCLUSÕES}

Compreendemos que efetivamente o APS é o caminho para levarmos O CP a quem necessita. É um desafio atender àqueles que precisam dos CP no final de suas vidas, promovendo a minimização da dor, conforto e dignidade do paciente, no entanto é prioridade, pois, reiteramos que nossos idosos aumentam a cada dia, no entanto, é fato a urgência pelo atendimento.

As políticas públicas de saúde possuem um longo caminho a trilhar para que sejam atendidos todos os pacientes que pela idade avançada, ou por DCNT precisam ter garantido o direito de receber o CP com brevidade. Muitas vezes a espera é longa e nem sempre chega a vez do paciente receber o CP em tempo.

A continuidade das discussões são necessárias, para que cada vitória nos leve mais próximos da determinação da OMS, que é ampliação do atendimento aos pacientes com necessidades de CP e assim entregar aos pacientes no fim da vida, um atendimento digno, justo, qualificado e humanizado.

É necessário a implantação de novas políticas públicas de saúde para que possamos ter na APS, um programa específico objetivando a sistematização do CP. Os recursos humanos que hoje possuimos são poucos e não garantem a tão discutida dignidade aos pacientes no final da vida.

Reconhecemos a necessidade de mais estudos para garantirmos um futuro otimista e assertivo. Futuras pesquisas em busca da garantia de um futuro melhor, fará do MF, um especialista comprometido, de fato com a causa do CPC junto aos nossos idosos.

\section{REFERÊNCIAS}

1. Instituto Brasileiro de Geografia e Estatística (IBGE). Indicadores sociodemográficos prospectivos para o Brasil 1991-2030. São Paulo: Arbeit Factory; 2006.

2. Instituto Brasileiro de Geografia e Estatística. Indicadores sociodemográficos e de saúde no Brasil (Coordenação de População e Indicadores Sociais). Rio de Janeiro: IBGE; 2009.

3. Côbo VA, Dal Fabbro AL, Parreira ACSP, Pardi F. Cuidados Paliativos na Atenção Primária à Saúde: perspectiva dos profissionais de saúde. Boletim Academia Paulista de Psicologia [Internet]. 2019 dez: 39(97):225-235.

4. Ribeiro JR, Poles K. Cuidados Paliativos: Prática dos Médicos da Estratégia Saúde da Família. Revista Brasileira de Educação Médica. 2019; 43(3):62-72.

5. Coelho Neto GC, Antunes VH, Oliveira A. A prática da Medicina de Família e Comunidade no Brasil: contexto e perspectivas. Cad de Saúde Pública. 2019;35(1):e00170917.

6. Souza HL, Zaboli ELCP, Paz CRP, Schveitzer MC, Hohl KG, Pessalacia JDR. Cuidados Paliativos na Atenção Primária à Saúde: considerações éticas. Revista Bioética. 2015 ago; 
23(2):349-59.

7. Hennnemann-Krause L, Freitas LA, Daflon PMN. Cuidados Paliativos e Medicina de Família e Comunidade: conceitos e interseções. Revista Hupe. 2016 jul.-set; 15(3):286-293.

8. Moraes SLF, Kairalla MC. Avaliação dos conhecimentos dos acadêmicos do curso de medicina sobre os cuidados paliativos em pacientes terminais. Einstein. 2010; 8(2):1627.

9. Gryschek G, Pereira EAL, Hidalgo G. Médicos de Família e Cuidados Paliativos: contribuições ao currículo baseado em competências. Rev Bras Med Fam Comunidade. 2020;15(42):2012. https://doi.org/10.5712/ rbmfc15(42)2012

10. Saito DYT, Zoboli ELCP. Cuidados Paliativos e a Atenção Primária à Saúde: scoping review. Revista Bioética. 2015; 23(3):593-607.

11. Azevedo C, Rates CMP, Pessalacia JDR, Mata LRF. Perspectivas para os Cuidados Paliativos na Atenção Primária à Saúde: estudo descritivo. ABEn. 2016 dez; 15(4):683-693.

12. Andrade HS, Alves MGM, Carvalho SR, Silva Junior AG. A formação discursiva da Medicina de Família e Comunidade no Brasil. Rev Bras Med Fam Comunidade. 2018:28(3):e280311.

13. Silva MLSR. O papel do profissional da Atenção Primária à Saúde em cuidados paliativos. Rev Bras Med Fam Comunidade. 2014 jan.-mar; 9(30):45-53.

14. Ernesto SA, Duarte ED. Cuidado Paliativo na Atenção Domiciliar: conhecimento, definição e elegibilidade na perspectiva do professional de saúde. XVI Coloquio Panamericano de Investigación en Enfermaria [Internet]. 2018 [acesso em 2020 nov 02]. Disponível em: http:// coloquioenfermeria2018.sld.cu/index.php/coloquio/2018/ paper/download/242/400

15.Cordeiro AM, Oliveira GM, Rentería JM, Guimarães CA, Grupo de Estudo de Revisão Sistemática do Rio de Janeiro. Revisão sistemática: uma revisão narrativa. Rev Col Bras Cir. 2007 nov.-dez; 34(5):428-431. https://doi. org/10.1590/S0100-69912007000600012
16. Ferenhof HA, Fernandes RF. Desmistificando a revisão de literatura como base para a redação científica: Método: SSF. R ABC: Biblioteconomia em Santa Catarina. 2016 ago; 21(3):550-563.

17. Rother ET. Revisão sistemática X revisão narrativa. Acta Paul Enferm. 2007 jun; 20(2):5-6. https://doi.org/10.1590/ S0103-21002007000200001 